\title{
Towards a Relativistically Correct Characterization of Counterstreaming Plasmas. I. Distribution Functions
}

\author{
M. Lazar ${ }^{*}$, A. Stockem ${ }^{2}$ and R. Schlickeiser ${ }^{1,3}$ \\ ${ }^{I}$ Research Department - Plasmas with Complex Interactions, Ruhr-Universität Bochum, D-44780 Bochum, Germany \\ ${ }^{2}$ GoLP/Inst. Plasmas e Fusão Nuclear, Instituto Superior Técnico, Lisbon, Portugal \\ ${ }^{3}$ Institut für Theoretische Physik, Lehrstuhl IV: Weltraum- und Astrophysik, Ruhr-Universität Bochum, D-44780 \\ Bochum, Germany
}

\begin{abstract}
The aim of this work is to provide a relativistically correct characterization for the stability of counterstreaming plasma structures ubiquitous in fusion plasma experiments and astrophysical sources of nonthermal radiation. Here, in the first part of this work, a new relativistically correct approach is formulated for the counterstreaming plasmas in thermal equilibrium, on the basis of the relativistic Jüttner-Maxwell distribution function and a correct representation of this distribution in the laboratory frame of reference by using the appropriate Lorentz transformations for momentum and energy. The particle velocity resulting from the thermal motion and the bulk displacement of plasma particles is thus limited according to the relativistic theory to less than $c$ (the speed of light in vacuum). New criteria are derived for the existence of counterstreams conditioned by the magnitude of their bulk velocity with respect to the thermal speed. Accurate simplified forms of the distribution functions derived here for different limits of the streaming velocity and the plasma temperature, will be invoked in the second part of this work as input to the stability analysis of these systems.
\end{abstract}

PACS Numbers: 52.25.Dg, 52.35.-g; 52.35.Qz; 52.27.-h; 52.27.Ny.

Keywords: Relativistic plasmas, beams, counterstreaming plasmas, high temperatures; analytical models, stability.

\section{INTRODUCTION}

Beam-plasma interactions have received great interest in both astrophysical and laboratory plasma applications. Plasma beams, shells and more or less collimated flows do seem to be a widespread presence on all size scales in space from extragalactic down to planetary [1]. With the present observational technique we are able to visualize intense charged beams in flares or coronal mass ejections of our Sun or further stars within our own galaxy, but also relativistic jets with speeds nearly equal to speed of light in quasars and radiogalaxies or from super-massive black holes at the centers of active galaxies. There is also indirect evidence for the existence of the energetic flows of plasma through nonthermal emissions incoming from space [2]. Signatures of charged particle beams include electromagnetic plasma emissions from bremsstrahlung to synchrotron radiation. The hard gamma-ray and x-ray spectrum of cosmic radiation are synchrotron emissions believed to originate in highly energetic collisions of relativistic beams with the widespread ionized matter. Thus, such relativistic jets have now been confirmed in astrophysical objects: weakly relativistic jets with a bulk Lorentz factor $\Gamma_{0}>1$ in microquasars [3], relativistic and ultrarelativistic jets $\left(\Gamma_{0}=5 \div 10^{3}\right)$ in active galactic nuclei (AGN) [4], and ultrarelativistic jets $\left(\Gamma_{0} \simeq 70 \div 300\right)$ in gamma ray bursts $(\mathrm{GRBs})[5]$.

*Address correspondence to this author at the Research Department Plasmas with Complex Interactions, Ruhr-Universität Bochum, D-44780 Bochum, Germany; Tel: +49 23432 23799; Fax: +49 23432 14177;

E-mail:mlazar@tp4.rub.de
Linear and nonlinear aspects of the beam-plasma interaction and instabilities with implications in plasma experiments and astrophysics have been reviewed in many textbooks [6-11]. Various theoretical models used for describing the stability properties of such beam-plasma systems include low- and high-density beams [6,9], highly energetic relativistic beams $[6,8]$, macroscopic fluid or kinetic treatments, linear models for the instability initiation $[6,8-10]$ or nonlinear models for the instability stabilization [7], finite dimensions and different shapes of the beam [6, $10]$, and also the effects of particle thermal spread, limited however to a classical nonrelativistic approach $[6,9]$.

Moreover, relevant for us here is the problem of return currents discussed in great detail in Ref. [11]. An imminent occurrence of countermoving streams in any beam-plasma system is proved, besides a considerably large lifetime of these counterstreams, whatever the mechanism of return current formation may be, e.g., the weak effect of magnetic induction or a displacement electric field. For example, an electric field builds up if a beam enters a surrounding plasma, and this electric field decelerates the particles of the beam and accelerates background electrons to form a return current $[11,12]$. In the absence of collisions (or other frictions) with background electrons and ions, the electric field becomes negligible and the opposite currents cancel each other and form what we call counterstreaming plasmas.

The plasma temperature also plays a significant role for the stability of astrophysical systems, where it can reach important values, e.g., for the solar corona $\left(T \sim 5 \cdot 10^{6} \mathrm{~K}\right.$, and the ratio $\left.\mu=m c^{2} / k_{B} T \sim 10^{3}\right)$, the sources of GRBs 
must be weakly-relativistic with $\left(k_{B} T \sim 1 \mathrm{keV}\right.$ [13] and $\mu \approx 50$ ), and the active galactic nuclei are fully relativistic plasmas with temperatures up to $10^{12} \mathrm{~K}$, i.e. $\mu<6 \cdot 10^{-3}$ [14].

In nonstreaming plasmas, a relativistically correct approach is important not only for high kinetic energies [15, 16], when the Lorentz factor of plasma particles becomes large enough, $\gamma=\left(1-v^{2} / c^{2}\right)^{-1 / 2} \gg 1$, and increases the particle mass $(m \rightarrow \gamma m)$, but also for the low energies of plasma particles, where the Lorentz factor does not intervene $(\gamma \simeq 1)$, but for a relativistically rigorous treatment one should limit the representation of the distribution functions of plasma particles to velocities less than the speed of light in vacuum, $v<c$. For plasmas at thermal equilibrium, such limitation is possible by modeling plasma particle velocity distribution with a relativistic Maxwellian introduced by Jüttner [17], see below in Sec. I, Eq. (1). By using a relativitistic restriction of the particle velocity to less than $c$, it has been rigorously shown that, in contrast to the classical theory of Landau, the so-called superluminal waves with phase velocities above $c(\omega / k>c)$ cannot resonate and exchange energy with plasma particles [18-26]. Moreover, recently, fully relativistic molecular dynamics simulation have reconfirmed the distribution function in the form proposed by Jüttner as the correct relativistic equilibrium velocity distribution [27].

For beam-plasma systems or counterstreaming plasmas (in thermal equilibrium), a relativistically correct approach requires not only a relativistic (Maxwellian) distribution function, but also a correct representation of this distribution in the laboratory frame of reference by using the appropriate Lorentz transformations for momentum and energy limiting the particle velocity resulting from the thermal motion and the bulk displacement of plasma particles to less than $c$. Treating counterstreaming plasma systems correctly relativistic is therefore not a simple task [28]. Plasma streams are described by the drift Jüttner distributions where different momentum components become coupled via the Lorentz factor and prohibit a simple analytical approach. Thus, simplified forms of the relativistic distributions derived with mean values of the thermal Lorentz factor, or in different limits of a low or a high temperature, and a slow or very energetic streaming motion, are frequently proposed to make the analysis tractable. However, the accuracy of the simplified representations is in many cases altered either by the restrictions used, or most often by the Lorentz transformations applied to the velocity (classical) and not to the momentum as required by the relativistic theory.

In this work we reconsider the relativistically correct characterization of the counterstreaming plasmas on the basis of such relativistic distribution function and the appropriate Lorentz transformations for momentum and energy. For clarity, we start with modeling nonstreaming plasmas in Sec. II, and then extend to symmetric and asymmetric counterstreams in Sec. III. Due to the thermal spread of plasma particles the existence of counterstreams is conditioned by the magnitude of their bulk velocity with respect to the thermal speed. A classification into different cases via the Lorentz factor of the beams is given, the criterium for the existence of counterstreams is derived and new simplified models of the counterstreaming plasmas are proposed with arguments. Discussions and conclusions of this first work are included in the last section.

\section{RELATIVISTIC NONSTREAMING PLASMAS}

The Jüttner (relativistic Maxwellian) distribution function is given by

$$
F_{p}=C \exp (-\mu \gamma)=C \exp \left[-\frac{m c^{2}}{k_{B} T}\left(1+\frac{p^{2}}{m^{2} c^{2}}\right)^{1 / 2}\right]
$$

where

$$
C(\mu)=\frac{\mu}{4 \pi(m c)^{3} K_{2}(\mu)}, \quad \mu=\frac{m c^{2}}{k_{B} T}
$$

$\gamma=\left(1-v^{2} / c^{2}\right)^{-1 / 2}$ is the Lorentz factor, $K_{2}(\mu)$ denotes the modified Bessel function, and the distribution function is normalized as $\int_{-\infty}^{+\infty} d^{3} p F_{p}=1$. This distribution function is displayed in Fig. (1) for four representative regimes of plasmas.

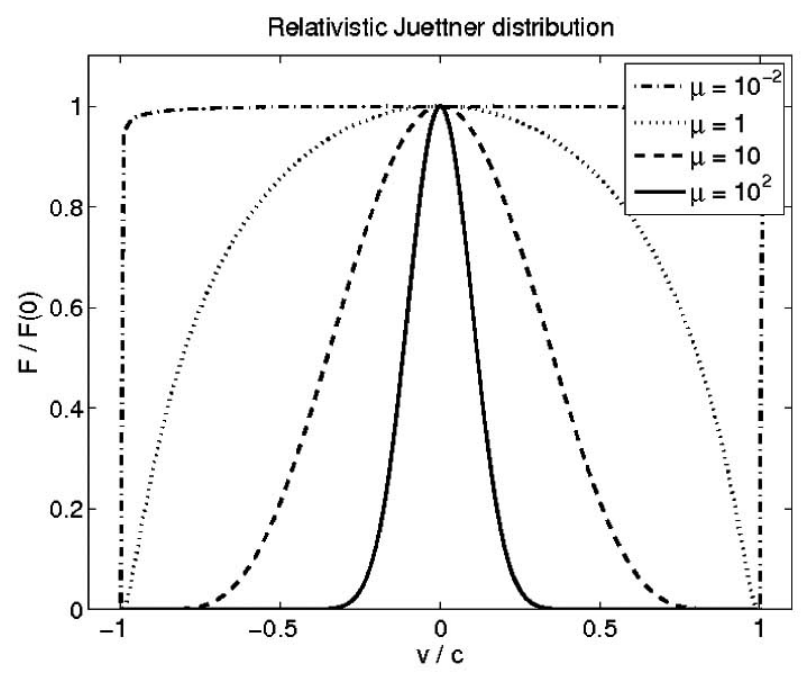

Fig. (1). Relativistic distribution function (1) in the particle velocity interval $(-c, c)$ for four representative cases: ultrarelativistic high temperatures $\left(\mu=10^{-2}\right)$, relativistic $(\mu=1)$, weakly relativistic temperatures $(\mu=10)$ and low plasma temperatures $\left(\mu=10^{2}\right)$.

Let introduce an universally accepted classification of plasmas according to their temperature (see Ref. [20] and Fig. (1) therein). Thus, in terms of the thermal parameter $\mu=m c^{2} / k_{B} T$ we can have plasmas with

- $\quad$ ultrarelativistic temperatures: $\mu \ll 1$,

- $\quad$ relativistic temperatures : $\mu \leqslant_{\sim}$,

- weakly (or mildly) relativistic temperatures: $\mu>1$, or

- low temperatures: $\mu \gg 1$, 
to which we can add the limits of

- the nonrelativistic classical treatment obtained for no limitation of plasma particle velocity, i.e., $c \rightarrow \infty$ (when also $\mu \rightarrow \infty$ ), and

- $\quad$ the cold plasma limit $(T \rightarrow 0)$ described by a Dirac distribution $f_{v} \sim \delta\left(v_{x}\right) \delta\left(v_{y}\right) \delta\left(v_{z}\right)$

In these limits, the plasma should be described by reduced forms of the Jüttner function (1), and below we proceed to their derivation, and then, for confirmation, these limit forms will be plotted and fitted with the relativistic distribution (1). Here we characterize only the electron plasma component but similar criteria can be attributed to the proton component as well (for ions, which are much heavier, it would probably be realistic to resume only to the weakly relativistic effects).

There is no convenient simplification for the distribution (1) in the limit of very high ultrarelativistic temperatures so as we turn and look to lower temperature approximations of this distribution function.

\subsection{Weakly Relativistic Limit}

A plasma with weakly relativistic temperature, i.e., $\mu>1$, is predominantly populated by low energetic electrons with small values for the Lorentz factor

$\gamma=\left(1+\frac{p^{2}}{m^{2} c^{2}}\right)^{1 / 2} \simeq 1+\frac{p^{2}}{2 m^{2} c^{2}}$,

and the momentum

$p=m \gamma v=m v\left(1-\frac{v^{2}}{c^{2}}\right)^{-1 / 2} \simeq m v\left(1+\frac{v^{2}}{2 c^{2}}\right)$.

By substituting (3) and (4) in (1) we find a weakly relativistic form of the distribution function

$F_{v} \simeq C \exp \left[-\frac{m c^{2}}{k_{B} T}\left(1+\frac{p^{2}}{2 m^{2} c^{2}}\right)\right]$

(a) Juettner (R) versus weakly relativistic (W)

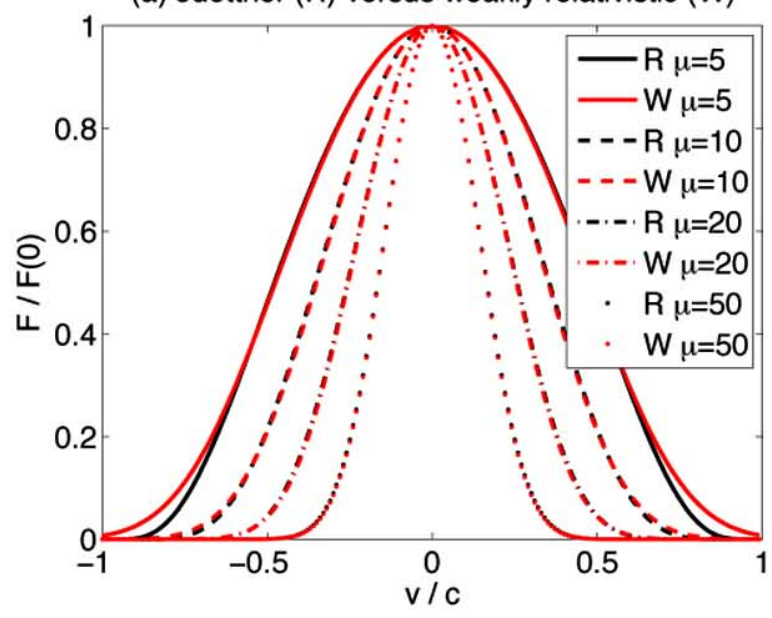

$\simeq C \exp (-\mu) \exp \left[-\frac{m v^{2}}{2 k_{B} T}\left(1+\frac{v^{2}}{c^{2}}\right)\right]$.

This is displayed in Fig. (2a) by comparison to the general form (1). Whether they fit well, the reduced form (5) can be used to describe plasmas at equilibrium and with weakly relativistic temperatures, i.e., $\mu \gtrsim 1$.

\subsection{Low Temperature Plasma}

At sufficiently low temperatures, we can define thermal velocity of plasma particles, $v_{T}=\sqrt{2 k_{B} T / m}$, which is much smaller than the speed of light in vacuum, $c$. In this case $\mu=m c^{2} / k_{B} T \gg 1$, the modified Bessel function $K_{2}(\mu \gg 1) \simeq(\pi /(2 \mu))^{1 / 2} \exp (-\mu)$, and the equilibrium distribution function (5) simplifies to a Maxwellian

$f_{v}=m^{3} F_{v}(\mu \gg 1)=\left(\frac{m}{2 \pi k_{B} T}\right)^{3 / 2} \exp \left[-\frac{m v^{2}}{2 k_{B} T}\left(1+\frac{v^{2}}{c^{2}}\right)\right]$.

The normalization constant is more accessible in this form, but the distribution is still shifted by the relativistic correction factor $\left(1+v^{2} / c^{2}\right)$, and, according to our assumption it is suitable only for sufficiently large $\mu \gg 1$, e.g., $\mu>10$.

\subsection{Nonrelativistic (Classic) Theory}

Transition to a nonrelativistic classic treatment requires $c \rightarrow \infty$ in (6) leading to the well known MaxwellBoltzmann distribution function

$$
f_{v}^{M B}=\lim _{c \rightarrow \infty} f_{v}=\left(\frac{m}{2 \pi k_{B} T}\right)^{3 / 2} \exp \left(-\frac{m v^{2}}{2 \pi k_{B} T}\right)=\frac{1}{\pi^{3 / 2} v_{T}^{3}} \exp \left(-\frac{v^{2}}{v_{T}^{2}}\right)
$$

which is normalized as $\int_{-\infty}^{+\infty} d^{3} v f_{v}=1$. According to Fig. (2b), the standard form (7) can be used at very low temperatures, i.e., very large $\mu \gtrsim 100$, since it approaches quite well the relativistically correct form (6).

\section{(b) Low-temp. (LT) versus nonrel. theory (N)}

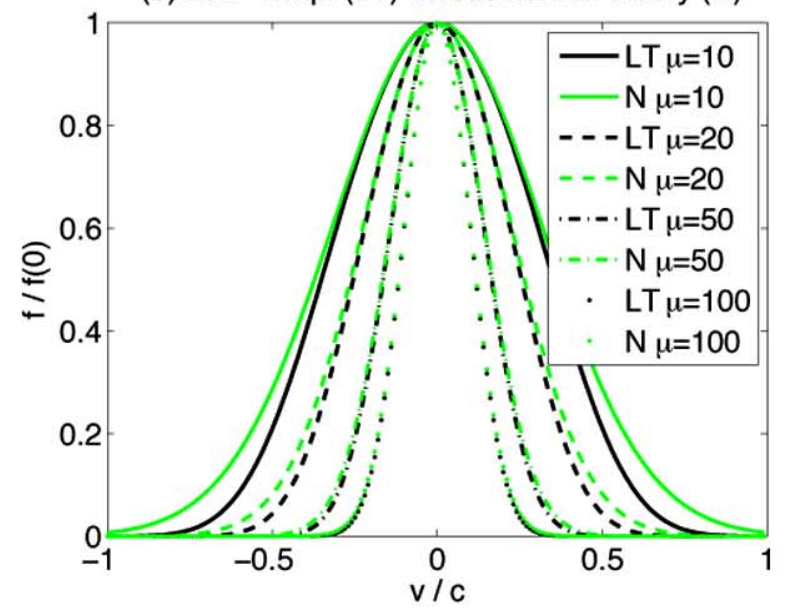

Fig. (2). (a) The relativistic Jüttner distribution (1) for weakly relativistic and nonrelativistic temperatures $(\mu>1)$ plotted in black and the weakly relativistic approximation (5) in red. (b) The low temperature approximation (6) plotted in black and the nonrelativistic approximation (7) in green. 


\subsection{Interlude}

We have shown that in a weakly relativistic regime with moderately high temperatures, the approximation (5) fits very well with the general distribution function (1). There is however another "weaker" approximation

$$
f_{v} \simeq C \exp (-\mu) \exp \left(-\frac{m v^{2}}{2 k_{B} T}\right)=C \exp (-\mu) \exp \left(-\frac{v^{2}}{v_{T}^{2}}\right),
$$

that is often used for weakly relativistic temperatures, probably because it is simpler than (5). But, according to Fig. (3), in the range of weakly relativistic temperatures, this is markedly deviated from plots of the approximation (5), and of course, from the exact relativistic Jüttner distribution function (1). Thus, the reduced form (5) remains more relevant for the weakly relativistic regime than the approximation (8).

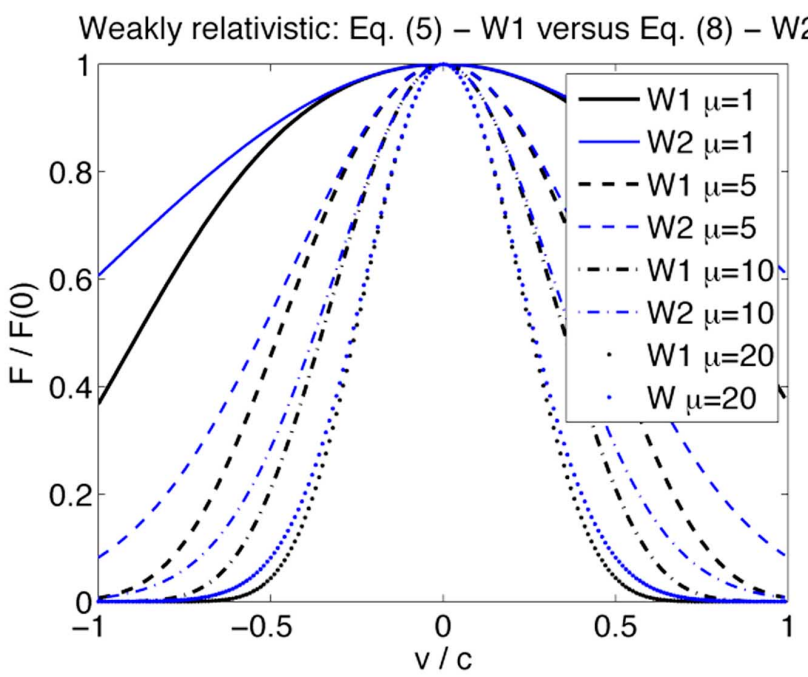

Fig. (3). Comparison of the approximations (5) and (8) for weakly relativistic temperatures $(\mu>1)$.

\section{COUNTERSTREAMING RELATIVISTIC PLASMAS}

Here we proceed to finding similar criteria and introduce more simple limit forms for the velocity distribution functions describing counterstreaming plasmas. For a streaming plasma we define a new kinetic parameter $\mu_{0}=m c^{2} / E_{c}=1 /\left(\Gamma_{0}-1\right)$ in terms of the bulk relativistic kinetic energy $E_{c}=m c^{2}\left(\Gamma_{0}-1\right)$ of particles, streaming with the speed $V_{0}$. Using this new parameter or the bulk Lorentz factor $\Gamma_{0}=\left(1-V_{0}^{2} / c^{2}\right)^{-1 / 2}$ the streams can be classified as follows:

1. ultrarelativistic streams for $\mu_{0} \ll 1$ that means $\Gamma_{0} \gg 2\left(V_{0} \rightarrow c\right)$,

2. relativistic streams for $\mu_{0} \leq 1$ that means $\Gamma_{0} \geq 2$ $\left(V_{0} \geq \sqrt{3} c / 2 \simeq 0.87 c\right)$,

3. weakly relativistic streams for $\mu_{0}>1$ that means $1<\Gamma_{0}<2\left(V_{0}<\sqrt{3} c / 2\right)$,
4. nonrelativistic streams for $\Gamma_{0} \simeq 1\left(V_{0} \ll c\right)$.

\subsection{Transformations to the Laboratory Frame. Symmetric Counterstreams}

In order to keep the analysis tractable, first we consider symmetric counterstreams with the same bulk velocity $V_{1}=\left|V_{2}\right|=V_{0} \quad$ (and the same Lorentz factor $\Gamma_{1}=\Gamma_{2}=\Gamma_{0} \equiv\left(1-V_{0}^{2} / c^{2}\right)^{-1 / 2}$, and, in their own frame at rest (script $R$ ), with the same density $n_{1}=n_{2}=n$ (and the plasma frequency $\omega_{p 1}=\omega_{p 2}=\omega_{p} \equiv 4 \pi n e^{2} / m$ ), the same temperature $T_{R 1}=T_{R 2}=T_{R}$ and a fully relativistic particle velocity distribution of the form (1)

$F_{p, 1}=F_{p, 2}=C_{R} \exp \left(-\mu_{R} \gamma_{R}\right)=C_{R} \exp \left[-\frac{m c^{2}}{k_{B} T_{R}}\left(1+\frac{p^{2}}{m^{2} c^{2}}\right)^{1 / 2}\right]$.

Here $C_{R}$ and $\mu_{R}$ take the same forms defined in (2). Two counterstreams are schematically shown in Fig. (4).

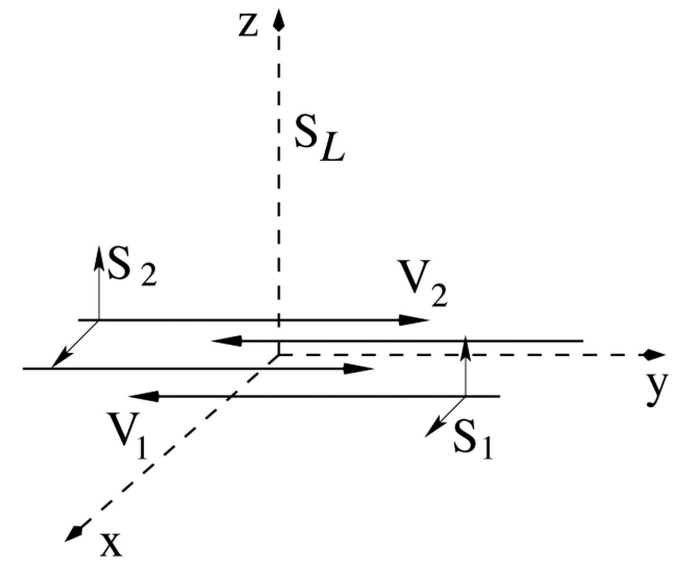

Fig. (4). Sketch of two symmetric counterstreaming plasmas, and their own frames $\mathrm{S}_{1,2}$ and the laboratory, $\mathrm{S}_{L}$ frame solidary to their mass center.

The bulk velocity of each stream, $V_{1,2}= \pm V_{0}$, is usually defined in the frame of background plasma. The analysis of such plasma systems simplifies if the observatory (or the laboratory frame, script $L$ in the next) is solidary with the mass center of the counterstreaming plasmas. Furthermore, for symmetric counterstreams the analysis will be limited, but it simplifies considerably allowing for a complete characterization that applies for any other frame where the streams can be assumed sufficiently symmetric.

Thus, we proceed to the transformation of the distributions $F_{p, 1}$ and $F_{p, 2}$ in the laboratory frame $\mathrm{S}_{L}$ by using the Lorentz transformations for energy

$\mathrm{S}_{1} \rightarrow \mathrm{S}_{L}$ (Fig. 4): $E_{R}=\Gamma_{0}\left(E_{L}-V_{0} p_{y}\right)$

$\mathrm{S}_{2} \rightarrow \mathrm{S}_{L}$ (Fig. 4) : $E_{R}=\Gamma_{0}\left(E_{L}+V_{0} p_{y}\right)$

or for the Lorentz factors, $\gamma=E /\left(m c^{2}\right)$, 
$\gamma_{R}=\Gamma_{0} \gamma_{L}\left(1-\frac{V_{0} v_{y}}{c^{2}}\right), \quad \gamma_{R}=\Gamma_{0} \gamma_{L}\left(1+\frac{V_{0} v_{y}}{c^{2}}\right)$.

The temperature is not invariant with Lorentz transformations, but changes as $T_{L}=T_{R} / \Gamma_{0}$ [28] leading to

$\mu_{L}=\Gamma_{0} \mu_{R}$.

Now the distributions (9) will transform as

$F_{p 1,2}^{L}=C_{L 1,2} \exp \left[-\mu_{L} \gamma_{L}\left(1 \mp \frac{V_{0} v_{y}}{c^{2}}\right)\right]$,

where the constants $C_{L 1,2}$ are given by the normalization of the distribution functions, $\int d^{3} p F_{p 1,2}^{L}=1$. For convenience, we omit the index $L$ in the next and use polar coordinates [23], $y \equiv p_{y} /(m c)=p_{\|} /(m c)$ and $\gamma \equiv \sqrt{1+\left(p_{\|}^{2}+p_{\perp}^{2}\right) /(m c)^{2}}$. The constant $C_{1,2}$ will be given by the normalization condition

$$
\begin{aligned}
& 1=\int d^{3} p F_{p 1,2}(\gamma, y)=2 \pi \int_{-\infty}^{\infty} d p_{\|} \int_{0}^{\infty} d p_{\perp} p_{\perp} F_{p 1,2}(\gamma, y) \\
& =2 \pi C_{1,2}(m c)^{3} \int_{1}^{\infty} d \gamma \gamma \exp (-\mu \gamma) \int_{-\sqrt{\gamma^{2}-1}}^{\sqrt{\gamma^{2}-1}} d y \exp \left( \pm \mu y \frac{V_{0}}{c}\right) \\
& =\frac{2 \pi C_{1,2}(m c)^{3}}{\mu V_{0} / c} \int_{1}^{\infty} d \gamma \gamma\left\{\exp \left[-\mu\left(\gamma \mp \sqrt{\gamma^{2}-1} \frac{V_{0}}{c}\right)\right]\right. \\
& \left.-\exp \left[-\mu\left(\gamma \pm \sqrt{\gamma^{2}-1} \frac{V_{0}}{c}\right)\right]\right\}
\end{aligned}
$$

Expanding the exponentials in power series, this condition yields

$$
C_{1}=C_{2}=\frac{\mu}{4 \pi(m c)^{3} I\left(\mu, \Gamma_{0}\right)},
$$

where the integral

$$
I\left(\mu, \Gamma_{0}\right)=\sum_{n=0}^{\infty}\left(\frac{\mu V_{0}^{2}}{2 c^{2}}\right)^{n} \frac{1}{\Gamma[n+1]} K_{n+2}(\mu)=\Gamma_{0}^{2} K_{2}\left(\mu / \Gamma_{0}\right)
$$

is calculated using the multiplication therorem [9.6.51] [29]. The normalization constants are equal and simplify as

$$
C_{s}=\frac{\mu}{4 \pi \Gamma_{0}^{2}(m c)^{3} K_{2}\left(\mu / \Gamma_{0}\right)},
$$

and the counterstreaming distribution function forms as

$$
F\left(\gamma, v_{y}\right)=\frac{C_{s}}{2}\left\{\exp \left[-\mu \gamma\left(1-\frac{V_{0} v_{y}}{c^{2}}\right)\right]+\exp \left[-\mu \gamma\left(1+\frac{V_{0} v_{y}}{c^{2}}\right)\right]\right\} .
$$

For a nonstreaming plasma $\left(V_{0}=0\right)$ the constant (16) reduces exactly to the normalization constant (2), $C_{s}\left(\mu, V_{0}=0\right)=C(\mu)$, and the counterstreaming distribution (17) transforms to the Jüttner distribution function (1). We should mention that the normalization constant $C_{s}$ from (16) can be obtained transforming the constant $C$ from (2) by using the general formalism developed in Ref. [28]: thermal parameter $\mu$ changes according to (11) and the elementary volume changes by contraction $d V_{L}=d V_{R} / \Gamma_{0}$ leading to an increasing of density $n_{L}=\Gamma_{0} n_{R}$ and an extra factor $\Gamma_{0}$ in the denominator of $C_{s}$.

\subsection{Criteria for the Existence of Symmetric Counter- streams}

For the sake of simplicity, here we keep considering symmetric counterstreams characterized by the same bulk velocity $V_{1}=V_{2}=V_{0}$ (and the same Lorentz factor $\left.\Gamma_{1}=\Gamma_{2}=\Gamma_{0} \equiv\left(1-V_{0}^{2} / c^{2}\right)^{-1 / 2}\right)$, the same temperature $T_{1}=T_{2}=T$, and described by the distribution function (17).

The distribution function $F(v)=F\left(v_{y}, v_{\perp}\right) \quad$ (where $\left.v_{y}^{2}+v_{\perp}^{2}=v^{2}\right)$ derived in (17) for two symmetric counterstreams propagating along the $y$-axis, shows two bumps only if the one-dimensional (slice) function $F(u)=F\left(v_{y}, v_{\perp}=0\right.$ ) (with $u:=v_{y} / c$ ) admits a minimum at $v_{y}=0$. For that, one has to prove conditions for a local minimum

(M1) the first derivative is zero: $F^{\prime}\left(v_{y}=0\right)=0$ (mandatory) and

(M2) the second derivative is positive: $F^{\prime \prime}\left(v_{y}=0\right)>0$ (sufficient).

To test these criteria, we look to the form (17) of the distribution function for two counterstreaming plasma beams, and define $U_{0}:=V_{0} / c, \quad \gamma_{\|}:=\gamma\left(v_{\perp}=0\right)=\gamma_{\|}(u)$, $F_{0}=F(u=0)=C_{s} \exp (-\mu)$ and

$N(u):=\frac{F(u)}{F_{0}}=\frac{1}{2} \exp (\mu)\left\{\begin{array}{l}\exp \left[-\mu \gamma_{\|}\left(1-u U_{0}\right)\right] \\ +\exp \left[-\mu \gamma_{\|}\left(1+u U_{0}\right)\right]\end{array}\right\}$

The first condition is always fulfilled:

$$
\begin{aligned}
& \left.\frac{d N}{d u}\right|_{u=0}=-\frac{\mu}{2} \exp (\mu)\left\{\left(u \gamma_{\|}^{3}\left(1-u U_{0}\right)-U_{0} \gamma_{\|}\right) \exp \left[-\mu \gamma_{\|}\left(1-u U_{0}\right)\right]\right. \\
& \left.+\left(u \gamma_{\|}^{3}\left(1+u U_{0}\right)+U_{0} \gamma_{\|}\right) \exp \left[-\mu \gamma_{\|}\left(1+u U_{0}\right)\right]\right\}\left.\right|_{u=0}=0
\end{aligned}
$$

The second condition needs to satisfy

$\left.\frac{d^{2} N}{d u^{2}}\right|_{u=0}=\left.\mu \gamma_{\|}^{2}\left(\mu U_{0}^{2}-\gamma_{\|}\right)\right|_{u=0}=\mu\left(\mu U_{0}^{2}-1\right)>0$,

that means

$\mu U_{0}^{2}>1$

and leading to the condition

$$
U_{0}=\frac{V_{0}}{c}>\sqrt{\frac{1}{\mu}} \text {. }
$$


This condition is not satisfied for hot plasmas with relativistic temperatures and a small thermal parameter $\mu \leq 1$ because the beam speed must be subluminal $V_{0}<c$. We immediately conclude that streams can not practically exist in such hot plasmas.

For less energetic but still (weakly) relativistic plasmas with $\mu \geqslant 1$, energetic streams can form with large bulk velocities given by (22), or with a Lorentz factor given by

$\Gamma_{0}>\sqrt{\frac{\mu}{\mu-1}}$.

If $\mu \simeq O(1)$, and $0<\mu-1 \ll 1$ the plasma beam must be relativistic with an extremely large speed $\left(V_{0} \rightarrow c\right)$ and Lorentz factor $\left(\Gamma_{0} \gg 1\right)$. Such relativistic jets with $\gamma_{0}=6 \div 40$ can be generated in AGN or, those with $\Gamma_{0} \simeq 70 \div 300$ are supposed to be at the origin of the afterglow synchrotron emissions in gamma-ray bursts [5].

In the case of a low-temperature plasma, $\mu \gg 1$, condition (22) guarantees the existence of streams with a speed that must exceed the thermal speed of plasma particles of the same species

$V_{0}>\frac{c}{\sqrt{\mu}}=\sqrt{\frac{k_{B} T}{m}} \simeq v_{T}$,

and a Lorentz factor $\Gamma_{0}>1$ (including less energetic nonrelativistic streams with $\left.\Gamma_{0} \geq 1\right)$.

\subsection{Arbitrary Counterstreams}

Here we generalize the analysis by considering two arbitrary counterstreams (schematically shown in Fig. 4) characterized by the bulk velocities $V_{1,2}$ and the bulk Lorentz factors $\Gamma_{1,2} \equiv\left(1-V_{1,2}^{2} / c^{2}\right)^{-1 / 2}$ in the laboratory frame. For each stream, in its own rest frame (subscript $R$ ), the thermal spread of plasma particles at equilibrium is described by the plasma temperature $T_{R 1,2}$, and a relativistic distribution function of the form (1)

$$
\begin{aligned}
& F_{p 1,2}=C_{R 1,2} \exp \left(-\mu_{R 1,2} \gamma_{R}\right) \\
& =C_{R 1,2} \exp \left[-\frac{m c^{2}}{k_{B} T_{R 1,2}}\left(1+\frac{p^{2}}{m^{2} c^{2}}\right)^{1 / 2}\right],
\end{aligned}
$$

where $C_{R}$ and $\mu_{R}$ keep the forms defined in (2).

Transformation to the laboratory frame $\mathrm{S}_{L}$ (in Fig. 4) yields for the energy

$\mathrm{S}_{1} \rightarrow \mathrm{S}_{L}$ (Fig. 4): $E_{R}=\Gamma_{1}\left(E_{L}-V_{1} p_{y}\right)$

$\mathrm{S}_{2} \rightarrow \mathrm{S}_{L}$ (Fig. 4): $E_{R}=\Gamma_{2}\left(E_{L}+V_{2} p_{y}\right)$

or for the Lorentz factors, $\gamma=E /\left(m c^{2}\right)$,
$\gamma_{R}=\Gamma_{1} \gamma_{L}\left(1-\frac{V_{1} v_{y}}{c^{2}}\right), \quad \gamma_{R}=\Gamma_{2} \gamma_{L}\left(1+\frac{V_{2} v_{y}}{c^{2}}\right)$,

and for the temperature $T_{L 1,2}=T_{R 1,2} / \Gamma_{1,2}$ [28] leading to

$\mu_{L 1,2}=\Gamma_{1,2} \mu_{R 1,2}$.

Using (26)--(27) the distributions (25) will change in the laboratory frame to

$F_{p 1,2}=C_{1,2} \exp \left[-\mu_{L 1,2} \gamma_{L}\left(1 \mp \frac{V_{1,2} v_{y}}{c^{2}}\right)\right]$,

where, similarly to equation (16), for the normalization constants we obtain

$C_{1,2}=\frac{\mu_{1,2}}{4 \pi \Gamma_{1,2}^{2}(m c)^{3} K_{2}\left(\mu_{1,2} / \Gamma_{1,2}\right)}$.

The counterstreaming distribution function then forms as

$F\left(\gamma, v_{y}\right)=C_{s}\left\{\begin{array}{l}\varepsilon_{1} \exp \left[-\mu_{1} \gamma\left(1-\frac{V_{1} v_{y}}{c^{2}}\right)\right] \\ +\varepsilon_{2} \exp \left[-\mu_{2} \gamma\left(1+\frac{V_{2} v_{y}}{c^{2}}\right)\right]\end{array}\right\}$,

where the intensity of each stream is defined as $\varepsilon_{1,2}=n_{1,2} /\left(n_{1}+n_{2}\right)=\omega_{p 1,2}^{2} /\left(\omega_{p 1}^{2}+\omega_{p 2}^{2}\right)$, and $\omega_{p 1,2}=4 \pi n_{1,2} e^{2} / m$ is the plasma frequency for each stream in part, and according to the normalization condition $\int_{-\infty}^{+\infty} d^{3} p F\left(\gamma, v_{y}\right)=1$, the normalization constant is

$C_{s}=\left(\frac{\varepsilon_{1}}{C_{1}}+\frac{\varepsilon_{2}}{C_{2}}\right)^{-1}$.

Moreover, if the laboratory frame is solidary with the mass center of the counterstreaming plasma system, the law of conservation for momentum yields the constraint

$\varepsilon_{1} \Gamma_{1} V_{1}=\varepsilon_{2} \Gamma_{2} V_{2}$.

This also provides the neutrality of the plasma system with a zero net current.

For a nonstreaming plasma $\left(V_{1,2}=0\right)$, the normalization constant (29) reduces exactly to $(2), C_{s}\left(\mu, V_{1,2}=0\right)=C(\mu)$, and the counterstreaming distribution (30) transforms to the Jüttner distribution function (1) for a plasma with two components of different densities and temperatures.

\subsection{Criteria for the Existence of Arbitrary Counter- streams}

The distribution function (30) for two arbitrary counterstreams shows two bumps only if there exists a minimum at $v_{y}=0$. Again, to test conditions (M1) and (M2) for a local minimum we use $u:=v_{y} / c, U_{1,2}:=V_{1,2} / c<1$, $\gamma_{\|}:=\gamma\left(v_{\perp}=0\right)=\gamma_{\|}(u)$, and transform the general form of the distribution function (30) to 
$F(u) \equiv F\left(\gamma_{\|}, u\right)=C_{s}\left\{\begin{array}{l}\varepsilon_{1} \exp \left[-\mu_{1} \gamma_{\|}\left(1-u U_{1}\right)\right] \\ +\varepsilon_{2} \exp \left[-\mu_{2} \gamma_{\|}\left(1+u U_{2}\right)\right]\end{array}\right\}$.

According to the first condition (M1) the first derivative must be zero

$$
\begin{aligned}
&\left.F^{\prime}(u)\right|_{u=0}=-C_{s} \mu_{1} \varepsilon_{1}\left[u \gamma_{\|}^{3}\left(1-u U_{1}\right)-U_{1} \gamma_{\|}\right] \\
& \times\left.\exp \left[-\mu_{1} \gamma_{\|}\left(1-u U_{1}\right)\right]\right|_{u=0} \\
&-\left.C_{s} \mu_{2} \varepsilon_{2}\left[u \gamma_{\|}^{3}\left(1+u U_{2}\right)+U_{2} \gamma_{\|}\right] \exp \left[-\mu_{2} \gamma_{\|}\left(1+u U_{2}\right)\right]\right|_{u=0}=0
\end{aligned}
$$

which yields

$\mu_{1} \varepsilon_{1} U_{1} \exp \left(-\mu_{1}\right)=\mu_{2} \varepsilon_{2} U_{2} \exp \left(-\mu_{2}\right)$.

The second condition (M2) applied to (30) needs to satisfy

$$
\begin{aligned}
\left.F^{\prime \prime}(u)\right|_{u=0}= & C_{s}\left[\mu_{1} \varepsilon_{1}\left(\mu_{1} U_{1}^{2}-1\right) \exp \left(-\mu_{1}\right)\right. \\
& \left.+\mu_{2} \varepsilon_{2}\left(\mu_{2} U_{2}^{2}-1\right) \exp \left(-\mu_{2}\right)\right]>0,
\end{aligned}
$$

and using (35) this condition simplifies as

$$
\frac{\mu_{1} U_{1}^{2}-1}{U_{1}}+\frac{\mu_{2} U_{2}^{2}-1}{U_{2}}>0
$$

or more simple

$$
\mu_{1} U_{2} U_{1}^{2}+\left(\mu_{2} U_{2}^{2}-1\right) U_{1}-U_{2}>0 \text {. }
$$

For symmetric counterstreams these conditions, (35) and (38), reduce exactly to those obtained above in (19) and (21), respectively.

If we consider the left-hand side of condition (38) as a quadratic function of $U_{1}=V_{1} / c$, we could look for a simple interpretation of this condition in two distinct cases. When one of the streams, for example, the second is sufficiently energetic, i.e., $U_{2}^{2}>1 / \mu_{2}=k_{B} T_{2} / m c^{2}$, the condition (38) demands for the first stream to have a bulk velocity

$$
\begin{aligned}
& U_{1}>\frac{\mu_{2} U_{2}^{2}-1}{2 \mu_{1} U_{2}}\left\{\left[1+\frac{4 \mu_{1} U_{2}^{2}}{\left(\mu_{2} U_{2}^{2}-1\right)^{2}}\right]^{1 / 2}-1\right\} \\
& \simeq \frac{\mu_{2} U_{2}}{2 \mu_{1}}\left\{\left[1+\frac{4 \mu_{1}}{\mu_{2}^{2} U_{2}^{2}}\right]^{1 / 2}-1\right\},
\end{aligned}
$$

where in the last term $U_{2}^{2} \gg 1 / \mu_{2}$ has been used. Note, that this case applies only to nonrelativistic plasmas $\left(\mu_{2} \gg 1\right)$.

Here we identify two subcases. Thus, for $U_{2}^{2} \gg\left(4 \mu_{1} / \mu_{2}\right) / \mu_{2}$, a counterstreaming structure exist only for $U_{1}>1 /\left(\mu_{2} U_{2}\right)$. For $1 / \mu_{2}<U_{2}^{2} \gg\left(4 \mu_{1} / \mu_{2}\right) / \mu_{2}$ when $4 \mu_{1} \gg \mu_{2}$ (i.e., $T_{2} \gg T_{1} / 4$ ), the same condition (39) presumes $U_{1}>1 / \sqrt{\mu_{1}}=\left(k_{B} T_{1} / m c^{2}\right)^{1 / 2}$.

In the opposite case of a small bulk velocity satisfying $U_{2}^{2}<1 / \mu_{2}=k_{B} T_{2} / m c^{2}$, the existence condition (38) asks for

$$
\begin{aligned}
& U_{1}>\frac{1-\mu_{2} U_{2}^{2}}{2 \mu_{1} U_{2}}\left\{\left[1+\frac{4 \mu_{1} U_{2}^{2}}{\left(1-\mu_{2} U_{2}^{2}\right)^{2}}\right]^{1 / 2}+1\right\} \\
& \simeq \frac{1}{2 \mu_{1} U_{2}}\left\{\left[1+4 \mu_{1} U_{2}^{2}\right]^{1 / 2}+1\right\} .
\end{aligned}
$$

We find again two subcases, one for $U_{2}^{2} \ll 1 /\left(4 \mu_{1}\right)$, when the condition for the existence of the counterstreams becomes $U_{1}>1 /\left(\mu_{1} U_{2}\right)$, and if $1 /\left(4 \mu_{1}\right)<1 /\left(2 \mu_{1}\right) \ll U_{2}^{2}<1 / \mu_{2}$, the same condition imposes $U_{1}>U_{2}$.

\subsection{Reduced Forms of the Counterstreaming Distri- bution}

Now we proceed to the characterization of the counterstreaming plasma system in different energetic limits with reduced forms of the fully relativistic distribution function. Again, for a simple analysis, we limit to symmetric counterstreams described by the relativistically correct distribution function (17) and the existence condition (21).

\subsubsection{Ultrarelativistic Streams}

For ultrarelativistic streams with a very large bulk Lorentz factor $\Gamma_{0} \gg 2$, the bulk velocity approaches the speed of light $V_{0} \rightarrow c$ and the counterstreaming distribution (17) becomes independent of $V_{0}$

$F\left(\gamma, v_{y}\right)=\frac{C_{s}}{2}\left\{\exp \left[-\mu \gamma\left(1-\frac{v_{y}}{c}\right)\right]+\exp \left[-\mu \gamma\left(1+\frac{v_{y}}{c}\right)\right]\right\}$.

The normalization constant $C_{S}$ is given by (16). Moreover, if the Lorentz factor is sufficiently large

$\Gamma_{0} \gg(1+\sqrt{5}) / 2>1$,

and the plasma thermal factor is in the interval

$1 \simeq \Gamma_{0}^{2} /\left(\Gamma_{0}^{2}-1\right)<\mu \ll \Gamma_{0}$,

satisfying condition (21), or (22), for the existence of counterstreams, then the argument of the modified Bessel function in $C_{s}$ is very small, $\mu / \Gamma_{0} \ll 1$, and the modified Bessel function simplifies as $K_{2}\left(\mu / \Gamma_{0}\right) \simeq 2 \Gamma_{0}^{2} / \mu^{2}$, leading to a new form of the normalization constant

$$
C_{s} \simeq \frac{\mu^{3}}{8 \pi \Gamma_{0}^{4}(m c)^{3}}
$$

Plasma temperature can take any value, from nonrelativistic ( $\mu \gg 1)$ to weakly relativistic $(\mu>1)$, but according to (43) it is limited by condition $\mu \ll \Gamma_{0}$.

In Fig. (5) the ultrarelativistic approximation (41) is compared to the general relativistic form (17). In the ultrarelativistic case, the distribution function is independent of the initial beam speed $V_{0}$. If the condition for ultrarelativistic Lorentz factors $\Gamma_{0} \gg 2$ is fulfilled, (41) is a good approximation because plots of the exact distribution function only slightly varies on the outer bounds. 


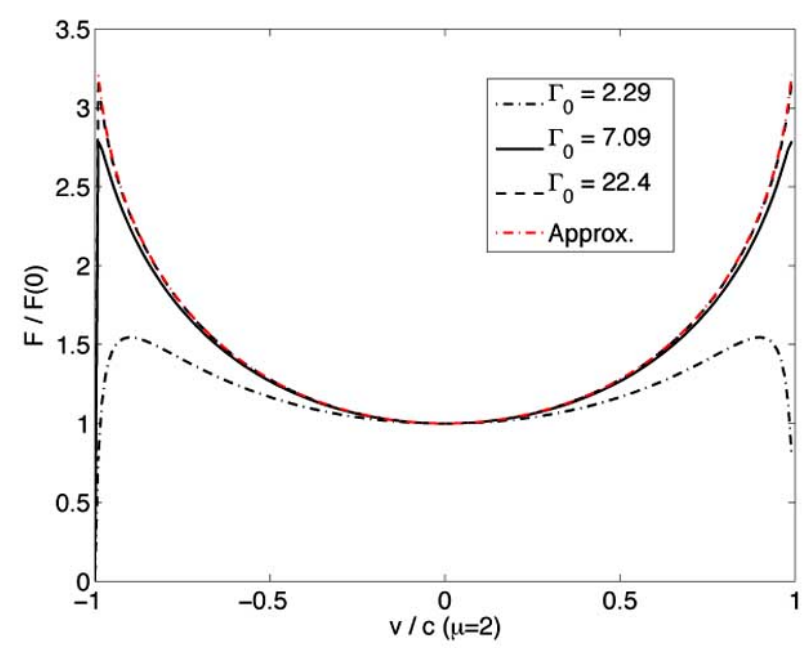

Fig. (5). Relativistic exact distribution function (17) for three different Lorentz factors $\Gamma_{0}=2.29, \Gamma_{0}=7.09, \Gamma_{0}=22.4$ plotted in black and the ultrarelativistic approximation (41) in red, which is independent of the streaming velocity.

\subsubsection{Low Temperature Plasmas}

In the opposite case of a large thermal factor, $\mu \gg \Gamma_{0}>1$, the plasma has a low temperature, the modified Bessel function simplifies asymptotically as

$K_{2}\left(\mu / \Gamma_{0} \gg 1\right) \simeq \sqrt{\frac{\pi \Gamma_{0}}{2 \mu}} \exp \left(-\frac{\mu}{\Gamma_{0}}\right)$

and the normalization constant (16) becomes

$C_{s} \simeq \frac{\mu^{3 / 2} \exp \left(\mu / \Gamma_{0}\right)}{(2 \pi)^{3 / 2} \Gamma_{0}^{5 / 2}(m c)^{3}}$.

The low temperature plasmas will be predominantly populated by electrons with a small momentum with respect to the mean steady-state flow momentum $p_{0}$, so that, we can again use the series representation for energy (or the Lorentz factor) and keep only terms up to the second order

$$
\begin{aligned}
& E(p) \equiv m c^{2}\left(1+\frac{p^{2}}{m^{2} c^{2}}\right)^{1 / 2}=m \gamma c^{2} \\
& \simeq \frac{m c^{2}}{\Gamma_{0}} \pm V_{0} p_{y}+\frac{p_{\perp}^{2}}{2 m \Gamma_{0}}+\frac{\left(p_{y} \mp p_{0}\right)^{2}}{2 m \Gamma_{0}^{3}} .
\end{aligned}
$$

here, " \pm " (and " $\mp "$, respectively, in the last term) correspond to the oppositely moving streams, i.e., the first and the second terms, respectively, in the distribution function (17). By substituting (46) and (47) the distribution function (17) becomes

$$
\begin{aligned}
& f_{p} \equiv m^{3} F_{v}\left(\mu \gg \Gamma_{0}\right) \\
& \simeq \frac{\mu^{3 / 2}}{2(2 \pi)^{3 / 2} \Gamma_{0}^{5 / 2} c^{3}} \exp \left(-\frac{\mu p_{\perp}^{2}}{2 m^{2} c^{2} \Gamma_{0}}\right) \\
& \times\left\{\exp \left[-\frac{\mu\left(p_{y}-p_{0}\right)^{2}}{2 m^{2} c^{2} \Gamma_{0}^{3}}\right]+\exp \left[-\frac{\mu\left(p_{y}+p_{0}\right)^{2}}{2 m^{2} c^{2} \Gamma_{0}^{3}}\right]\right\} .
\end{aligned}
$$

Further simplification can be obtained developing again the Lorentz factor $\gamma$ (in $p_{y}$ ) after $v_{y}$ around $V_{0}$

$$
\gamma\left(v_{y}\right) \equiv\left(1-\frac{v^{2}}{c^{2}}\right)^{-1 / 2} \simeq\left(1-\frac{v_{y}^{2}}{c^{2}}\right)^{-1 / 2} \simeq \Gamma_{0}+\left(v_{y} \mp V_{0}\right) \frac{V_{0}}{c^{2}} \Gamma_{0}^{3},
$$

and writing

$$
\begin{aligned}
& p_{y} \mp p_{0}=m\left(\gamma v_{y} \mp \Gamma_{0} V_{0}\right)=m \Gamma_{0}\left(v_{y} \mp V_{0}\right)+m v_{y}\left(v_{y} \mp V_{0}\right) \frac{V_{0}}{c^{2}} \Gamma_{0}^{3} \\
& \simeq m \Gamma_{0}\left(v_{y} \mp V_{0}\right)+m\left(v_{y} \mp V_{0}\right) \frac{V_{0}^{2}}{c^{2}} \Gamma_{0}^{3}=m\left(v_{y} \mp V_{0}\right) \Gamma_{0}^{3},
\end{aligned}
$$

where, according to the low temperature assumption, $v_{y}$ is sufficiently close to $V_{0}$. Now, by using (50), the distribution function which describes counterstreaming plasmas with low temperatures takes the form

$$
\begin{aligned}
& f_{v}=\frac{1}{2(\pi)^{3 / 2} v_{T}^{3} \Gamma_{0}^{5 / 2}} \exp \left(-\frac{v_{\perp}^{2}}{v_{T}^{2} \Gamma_{0}}\right) \\
& \times\left\{\exp \left[-\frac{\left(v_{y}-V_{0}\right)^{2} \Gamma_{0}^{3}}{v_{T}^{2}}\right]+\exp \left[-\frac{\left(v_{y}+V_{0}\right)^{2} \Gamma_{0}^{3}}{v_{T}^{2}}\right]\right\},
\end{aligned}
$$

where $v_{T}=\left(2 k_{B} T / m\right)^{1 / 2}$ is the thermal velocity introduced first time in Eq. (7).

For weakly relativistic streams $\left(1<\Gamma_{0}<2\right)$, this distribution function can be written exclusively in terms of the bulk velocity $V_{0}$ by using the approximations $\Gamma_{0} \simeq 1+V_{0}^{2} /\left(2 c^{2}\right), \Gamma_{0}^{-1} \simeq 1-V_{0}^{2} /\left(2 c^{2}\right), \Gamma_{0}^{3} \simeq 1+3 V_{0}^{2} /\left(2 c^{2}\right)$ and $\Gamma_{0}^{-5 / 2} \simeq 1-5 V_{0}^{2} /\left(4 c^{2}\right)$. For nonrelativistic streams, $\Gamma_{0} \simeq 1$ and the distribution function (48) reduces to the wellknown classic form of a Maxwellian counterstreaming distribution.

In Fig. (6) we display the low temperature approximation (48) and the exact distribution (17) for two different values of plasma temperature. In the case of a highly nonrelativistic plasma (Fig. 6b) the approximation fits well over the whole range of particle velocities. For a low $\mu$ (Fig. 6a) the approximation still reproduces the peaks and the shape of the bumps.

\section{CONCLUSIONS}

The main aim of this work is to provide a relativistically correct characterization for the stability of counterstreaming plasma structures ubiquitous not only in fusion plasma experiments but also in astrophysical sources where the observed nonthermal cosmic radiation originates. Here in the first part we have refined the relativistic models of the counterstreaming plasmas on the basis of the relativistic Maxwellian distribution function and the appropriate Lorentz transformations for momentum and energy. Counterstreaming plasma structures lead to the onset of plasma wave instabilities that will make the object of our investigations in the second part of this work. 

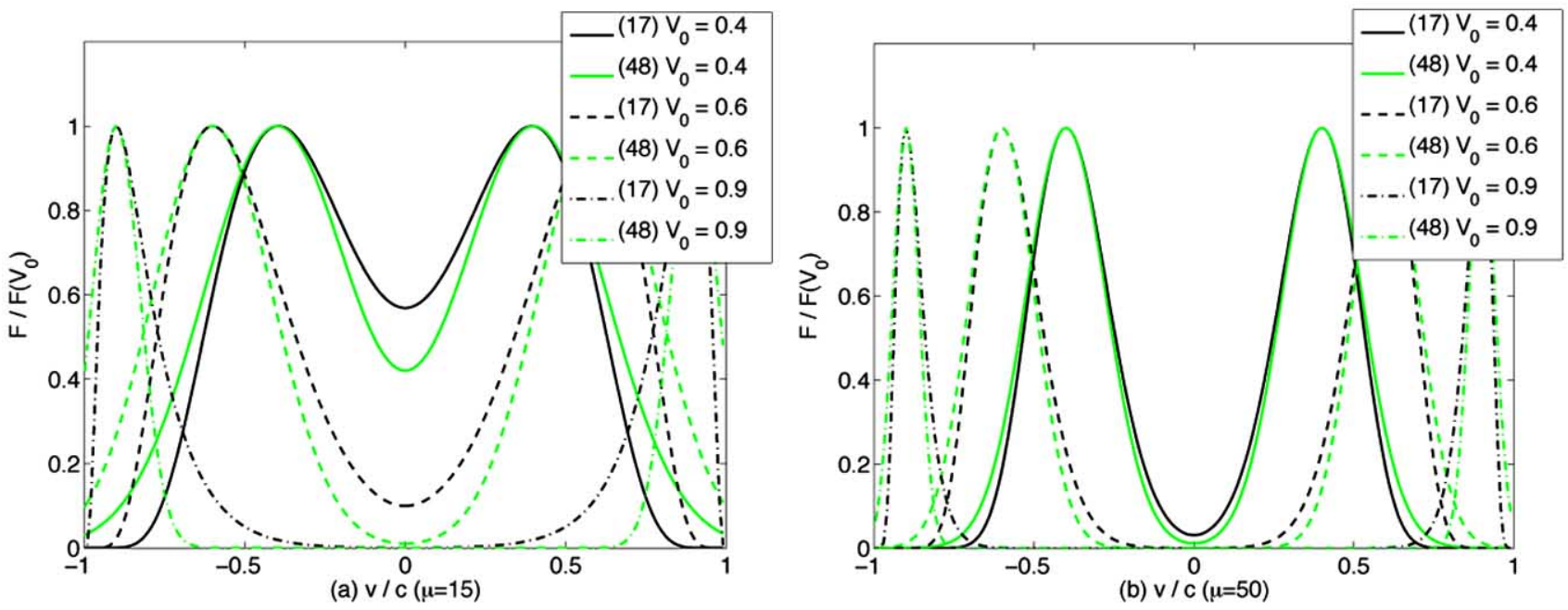

Fig. (6). Relativistic correct distribution function (17) for three different beam velocities $V_{0}=0.4, V_{0}=0.6, V_{0}=0.9$ and low plasma temperature given by (a) $\mu=15$, (b) $\mu=50$ plotted in black and the approximation (48) in green.

We have derived new criteria for the existence of symmetric or arbitrary counterstreams: due to the thermal spread of plasma particles the existence of counterstreams is conditioned by the magnitude of their bulk velocity with respect to the thermal speed. A new classification via the Lorentz factor of the beams has been introduced. Based on these rigorous criteria, the distribution functions have been simplified according to different limit cases of a cold plasma or plasmas with non-, weakly- or ultra-relativistic temperatures, combined with non-, weakly-, or ultrarelativistic bulk velocities of the streaming plasmas. The range of application is identified for each case in part by fitting the reduced forms with the exact fully relativistic distribution function. Such simple but relativistically correct representation of the counterstreaming distribution functions creates premises for developing new and accurate analytical approaches for studying their dispersion properties and stability.

\section{ACKNOWLEDGEMENTS}

ML acknowledges support from the Research Department - Plasmas with Complex Interactions, RuhrUniversität Bochum. RS acknowledges support from the Deutsche Forschungsgemeinschaft through grants Schl 201/19-1 and Schl 201/21-1.

\section{REFERENCES}

[1] Brown JC. Beams and jets in astrophysical plasmas. Plasma Phys Control Fusion 1985; 27: 1359-68.

[2] Ferrari A. Modeling Extragalactic Jets. Annu Rev Astron Astrophys 1998; 36: 539-98.

[3] Mirabel IF, Rodriguez LF. A superluminal source in the Galaxy. Nature 1994; 371: 46-8.

[4] Biretta JA, Sparks WB, Macchetto F. Hubble Space Telescope Observations of Superluminal Motion in the M87 Jet. Astrophys J 1999; 520: 621-6.

[5] Panaitescu A, Kumar P. Properties of relativistic jets in gamma-ray burst afterglows. Astrophys J 2002; 571: 779-89.

[6] Briggs RJ. Two-stream instabilities. In: Simon A, Thomson WB, Eds. Advances in Plasma Physics. New York: John Wiley \& Sons, Inc. 1971; vol. 4: p. 43.
[7] Davidson RC. Methods in nonlinear plasma theory. New York: Academic Press 1972

[8] Davidson RC. Theory of nonneutral plasma. Benjamin WA, Inc. 1974.

[9] Davidson RC. Kinetic waves and instabilities in a uniform plasma. In: Rosenbluth MN, Sagdeev RZ, Eds. Handbook of Plasma Physics. Amsterdam: North-Holland 1983; vol. 1: p. 519

[10] Humphries S. Charged Particle Beams. New York: John Wiley \& Sons, Inc. 1990.

[11] Benz AO. Plasma Astrophysics - Kinetic Processes in Solar and Stellar Coronae. Dorddrecht: Kluwer Academic Publishers 1993.

[12] Karlicky M. Electron Beam-Plasma Interaction and the ReturnCurrent Formation. Astrophys J 2009; 690: 189-97.

[13] Piran T. The physics of gamma-ray bursts. Rev Mod Phys 2004; 76: 1143-210.

[14] Urry CM, Padovani P. Unified schemes for radio-loud active galactic nuclei. Publ Astron Soc Pac 1995; 107: 803-45.

[15] Stockem A, Lerche I, Schlickeiser R. The relativistic filamentation instability in magnetized plasmas. Astrophys J 2007; 659: 419-25.

[16] Medvedev M, Loeb A. Generation of magnetic fields in the relativistic shock of gamma-ray burst sources. Astrophys J 1999; 526: 697-706.

[17] Jüttner F. Das Maxwellsche Gesetz der Geschwindigkeitsverteilung in der Relativtheorie. Ann Phys 1911; 339: 856-82.

[18] Swanson DG. Plasma Waves. New York: Academic Press 1989.

[19] Schlickeiser R. Longitudinal oscillations in hot isotropic Maxwellian plasmas. Phys Plasmas 1994; 1: 2119-24.

[20] Fichtner H, Schlickeiser R. On Landau damping in hot equilibrium plasmas. I. Longitudinal oscillations along an external magnetic field. Phys Plasmas 1995; 2: 1063-72.

[21] Schlickeiser R, Kneller M. Relativistic kinetic theory of waves in isotropic plasmas. J Plasma Phys 1997; 57: 709-40.

[22] Lazar M, Schlickeiser R. Relativistic kinetic theory of electromagnetic waves in equilibrium magnetized plasma. General dispersion equations. Can J Phys 2003; 81: 1377-87.

[23] Schlickeiser R. Covariant kinetic dispersion theory of linear waves in anisotropic plasmas. I. General dispersion relations, biMaxwellian distributions and nonrelativistic limits. Phys Plasmas 2004; 11: 5532-46.

[24] Lazar M, Schlickeiser R. Relativistic kinetic dispersion theory of linear parallel waves in magnetized plasmas with isotropic thermal distributions. New J Phys 2006; 8: 66-84.

[25] Lazar M, Schlickeiser R. Covariant kinetic dispersion theory of linear transverse waves parallel propagating in magnetized plasmas with thermal anisotropy. Phys Plasmas 2006; 13: 012110.

[26] Tautz RC, Schlickeiser R. Counterstreaming magnetized plasmas. I. Parallel wave propagation. Phys Plasmas 2005; 12: 122901. 
[27] Cubero D, Casado-Pascual J, Dunkel J, Talkner P, Hänggi P. Thermal equilibrium and statistical thermometers in special relativity. Phys Rev Lett 2007; 99: 170601.
[28] Wright TP, Hadley GR. Relativistic distribution functions and applications to electron beams. Phys Rev A 1975; 2: 686-97.

[29] Abramowitz M, Stegun IA. Handbook of mathematical functions. Washington: National Bureau of Standards 1972.

(C) Lazar et al.; Licensee Bentham Open.

This is an open access article licensed under the terms of the Creative Commons Attribution Non-Commercial License (http://creativecommons.org/licenses/ by$\mathrm{nc} / 3.0 /$ ) which permits unrestricted, non-commercial use, distribution and reproduction in any medium, provided the work is properly cited. 